\title{
RepGene: A Spreadsheet Template for the Management of Reporter Gene Assays
}

BioTechniques 30:1294-1298 (June 2001)

\author{
Patrick Y. Muller ${ }^{1}$, Luca \\ Barella ${ }^{1}$, and André $R$. \\ Miserez $^{1,2}$ \\ ${ }^{1}$ University of Basel and ${ }^{2}$ Basel \\ University Clinics, Basel, \\ Switzerland
}

\begin{abstract}
One of the most powerful techniques in molecular biology is the controlled expression of specific proteins by transfection of eukaryotic cells. This method has become feasible and highly sensitive and, thus, suitable for high-throughput reporter gene assays in basic and applied research. Moreover, the limiting factors are neither the transfection efficiency nor the functional analysis, but rather the ability to manage complex experimental protocols when multiple genes are co-transfected and/or when the effects of several chemical compounds are investigated within the same experiment. Here, we describe an easy-to-use and highly flexible spreadsheet template intended to rationalize and expedite the organization and data management of multi-step reporter gene assays. The objectives of this spreadsheet template are the design of the transfection protocol, the coordination of the administration of test compounds, and the graphical presentation and statistical analysis of the results.
\end{abstract}

\section{INTRODUCTION}

The regulation of gene expression is generally mediated by intracellular signal transduction pathways $(5,7)$. In the dissection of these mechanisms, reporter gene assays play a key role (1). The transcriptional activation of a gene of interest can be investigated by cloning a specific transcription factor response element, in combination with a reporter gene that encodes an easily detectable reporter protein. Most reporter genes encode enzymes catalyzing the conversion of specific substrates, enabling colorimetric, fluorometric, or luminometric detection (3). The variety of available reporter genes covers a broad range of applications, including the analysis of gene expression as part of signaling pathways, the functional analysis of regulatory sequences, the functional analysis of gene mutations, the temporal and spatial recording of gene expression, and the investigation of specific molecules regarding gene activation [i.e., drug screening (2)].

When multiple genes are co-transfected and/or when the effects of several chemical compounds are investigated within the same experiment, the flexible data management of complex reporter gene assays is a challenge. Currently, the limiting factors for an increase in productivity are neither the transfection efficiency nor the detection of the reporter protein, but rather the efficient coordination and data management of multi-step reporter gene experiments due to the lack of commercially available software. Although the detailed analysis requirements of reporter gene assays vary depending on the par- ticular application, the evaluation of the raw data is crucial for all reporter gene assays, especially if performed on a high-throughput scale. Usually, the data are recorded by a computer connected to the optical analysis instrument (e.g., spectrometer, fluorometer, or luminometer) that performs the readout of the reporter gene expression. If transient transfection is used, the expression of the reporter gene generally needs to be corrected for transfection efficiency. This is accomplished by cotransfection with a normalization reporter gene [e.g. Renilla reniformis luciferase (6)]. The so-called relative expression is then calculated as the quotient of the reporter gene expression values divided by the normalization expression values. For reasons of reproducibility, reporter gene assays are usually performed in duplicate within the same experiment, whereas the corresponding standard errors can be calculated as an intra-experimental quality control. Another important and frequently needed calculation is the subtraction of a specific baseline expression value from all other expression values. In addition, it is often needed to express the reporter gene expression values in relation to the expression value of a specific control sample. All these calculations are routinely performed by RepGene.

The RepGene spreadsheet template presented here has been developed to manage and expedite the entire experimental process. This includes the planning of the transfections, the potential administration of test compounds, and the recording, storage, and analysis of the data. 


\section{RESULTS AND DISCUSSION}

\section{Structure, Features, and Characteristics of RepGene}

RepGene is implemented on the basis of a Microsoft ${ }^{\circledR}$ Excel $^{\circledR}$ spreadsheet file and has been programmed in Visual Basic $^{\circledR}$ for Applications (VBA). Thus, it can be run on any system where Excel (version 97 or later for Microsoft Windows $^{\mathrm{TM}}$ and version 98 or later for Macintosh ${ }^{\circledR}$ ) has been installed. Structured worksheets guide the experimenter, step by step, through the entire experimental process. This comprises the general planning and culture media preparation, the setup of the cells and the transfection, data acquisition and evaluation, graphical presentation, and statistical analysis. The worksheets are organized in a logical and streamlined fashion, enabling a fast and structured performance of the experiments. Because of the modular assembly of RepGene, every worksheet can also be used and modified individually, providing the highest flexibility.

Every experiment is managed by a separate Excel file, which is structured into five interactive worksheets. On opening the file, a custom-made toolbar named "RepGene" appears, providing the user with the possibility of using several VBA programs for a variety of essential procedures. Every worksheet represents a specific experimental section and contains green-shaded cells for data entry (input variables provided by the user) and white cells containing output values calculated by RepGene (output variables).

Before starting the experiment, the Transfection worksheet should be com pleted to obtain an overview of the number of individual transfections and their characteristics. All five worksheets are formatted in the 96-well microplate format, but any other numbers and sizes of wells and culture dishes can be used equally. Another feature of RepGene is the tabular and schematic organization of the individual wells, their input values (i.e., parameters for the experimental setup), and their output values calculated from the acquired data. In each worksheet, all variables, either entered by the experimenter (green cells) or calculated by the software (white cells), are explained by pop-up windows, making RepGene easy to use in a self-explanatory way. Comments and explanations are provided where needed, and all cell formats and the formulae for the calculations can be independently modified by the user.

\section{Practical Use of RepGene}

The first worksheet to appear after opening the application is the Transfection worksheet, where the user is asked to enter all input variables concerning the transfection protocol (Figure 1). All input values, such as $(i)$ the stock concentrations of the reporter genes, (ii) the final volume of the transfection mixture in each well, (iii) the amounts of DNA to be transfected, and (iv) the ratios of DNA to the various solutions of the transfection kits, have their own input cells. The values of these cells can be independently modified for each of the wells of the cell culture dishes. The Transfection worksheet is configured as default for assays to be carried out in duplicate wells, preferably with the widely used lipofection technique (4). Alternatively, the transfections can be performed in Petri dishes; thus, it is possible to transfer the cells to the 96-well microplate after transfection. On the top of the Transfection worksheet, space is given for a short description of the ex- periment, which is then automatically taken over to all other worksheets in RepGene. In addition, the footer is preconfigured in all worksheets to display the printing date, time, and file name.

The second worksheet, called Media, guides the experimenter through the preparation of the culture media needed in different experimental phases. It therefore comprises the media before and, if needed, during and after the transfection and the wash and lysis buffers. The schematic representation is analogous for all three media and allows any compositions, concentrations, and volumes to be chosen. The setup of the cells in the culture dishes is another task that can be calculated by RepGene, on the basis of the number of cells and the volume needed in each well, the number of wells, and the cell concentration of the stock culture.

After transfection of the cells, various chemical compounds can be administered to investigate their influence on gene expression. The Compounds worksheet (which is optional) manages this experimental section. Its main function is the calculation of the volumes of stock solutions of the com pounds to be added to each well of the cell culture dish. Two independent sets of compounds, represented by set no. 1 and set no. 2 in the Compounds worksheet, can be simultaneously added to

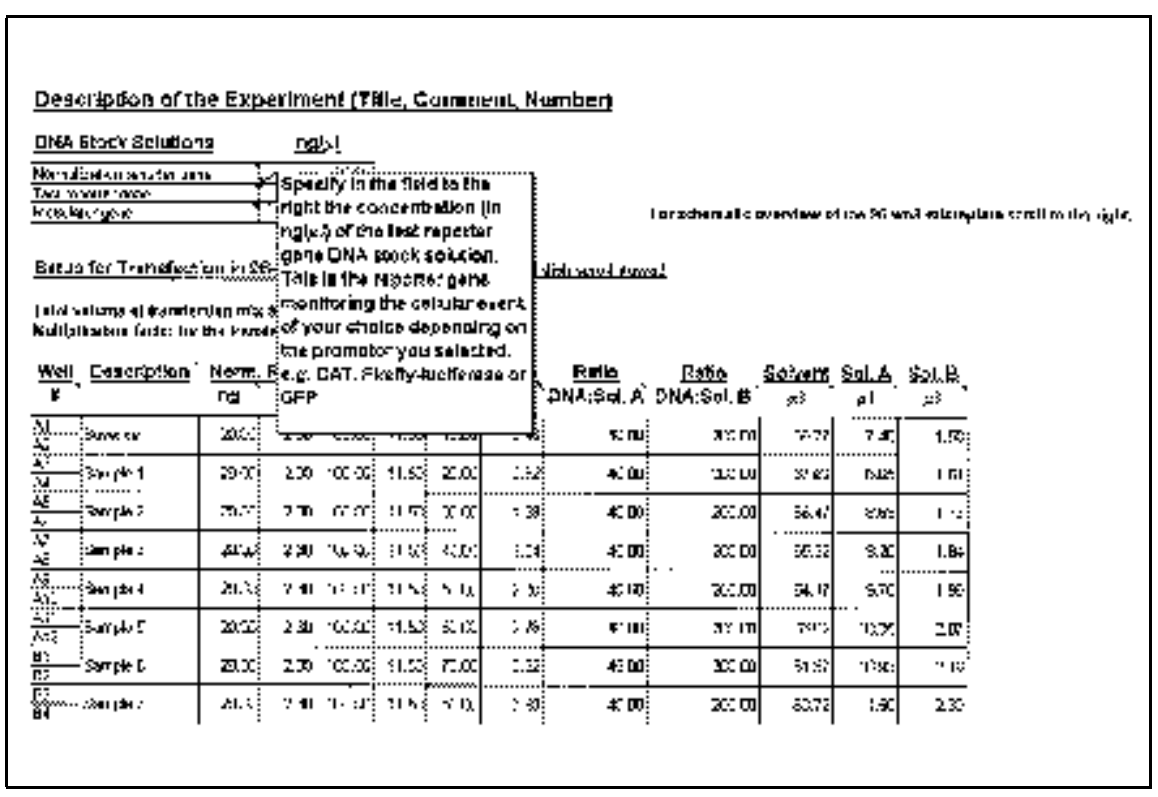

Figure 1. Screen output of the Transfection worksheet. All input variables concerning the transfection process can be entered in the green-shaded cells and are explained by pop-up windows. 


\section{}

each well. The input variables are the desired final concentrations of the com pounds; whereas, the cross-influence of the volume of set no. 1 on the volume of set no. 2, and vice versa, has been taken into consideration.

The last two worksheets are for the calculation, graphical presentation, and statistical analysis of the data generated by the experiment. The calculation and the graphical presentation are performed in the Analysis worksheet. Here, the expression values of the test reporter gene and the internal control reporter gene can either be entered manually by the user or imported, by a VBA program, from data files generated by analytical instruments, such as microplate fluorometers, luminometers, or spectrometers that are connected to computers. If the data files are opened with Excel, the fluorometric, luminometric, or absorption data are usually arranged according to the outline of a 96-well microplate ( 8 rows and 12 columns) and can therefore be automatically imported into the Analysis worksheet of RepGene. The first calculation performed by RepGene is the normalization of the reporter gene expression values, by dividing them by the expression values of the internal control reporter gene. As the use of an internal control reporter gene is not absolutely necessary in many experiments, all calculations are possible in the absence of an internal control reporter gene. The normalized or non-normalized expression values are represented by a 3-D chart, reflecting the outline of a 96-well microplate (Figure 2).

Subsequent calculations performed by RepGene comprise the averages of the expression values of the duplicates, their standard errors (arithmetically), the expression values with subtracted baseline, and the relative effect compared to a value of choice; the latter two are guided by a VBA program. These values are presented in an independent 2-D chart by columns and corresponding error bars. The descriptions of the columns are automatically taken over from the Transfection worksheet, where they have been typed in for the description of the wells of the microplate.

The statistical analysis can be performed using the corresponding menu item from the RepGene toolbar. In the optional Comparison worksheet, the reporter gene expressions can be com pared between two matched groups, namely group A and group B. These two groups can be interpreted as two different metabolic conditions of the cells, the absence or presence of an in- hibitor or inducer, two time points, two concentrations of compounds, and so on. In an alternating mode, every first duplicate of the experiment is automatically assigned to group A and every second duplicate to group B, respectively. This enables the calculation of ratios in group A divided by group B. To assess the significance level of differences between the two groups, it is possible, if the values follow a normal distribution, to perform a paired or an unpaired Student's $t$ test by selecting the corresponding items.

\section{CONCLUSION}

The RepGene spreadsheet template described here is a tool to manage com plex multi-step reporter gene assays on a high-throughput scale. It considerably expedites and rationalizes the experimental setup, data analysis, and data management while ensuring the highest reproducibility. Since every experiment is tracked by a separate Excel file, auto-

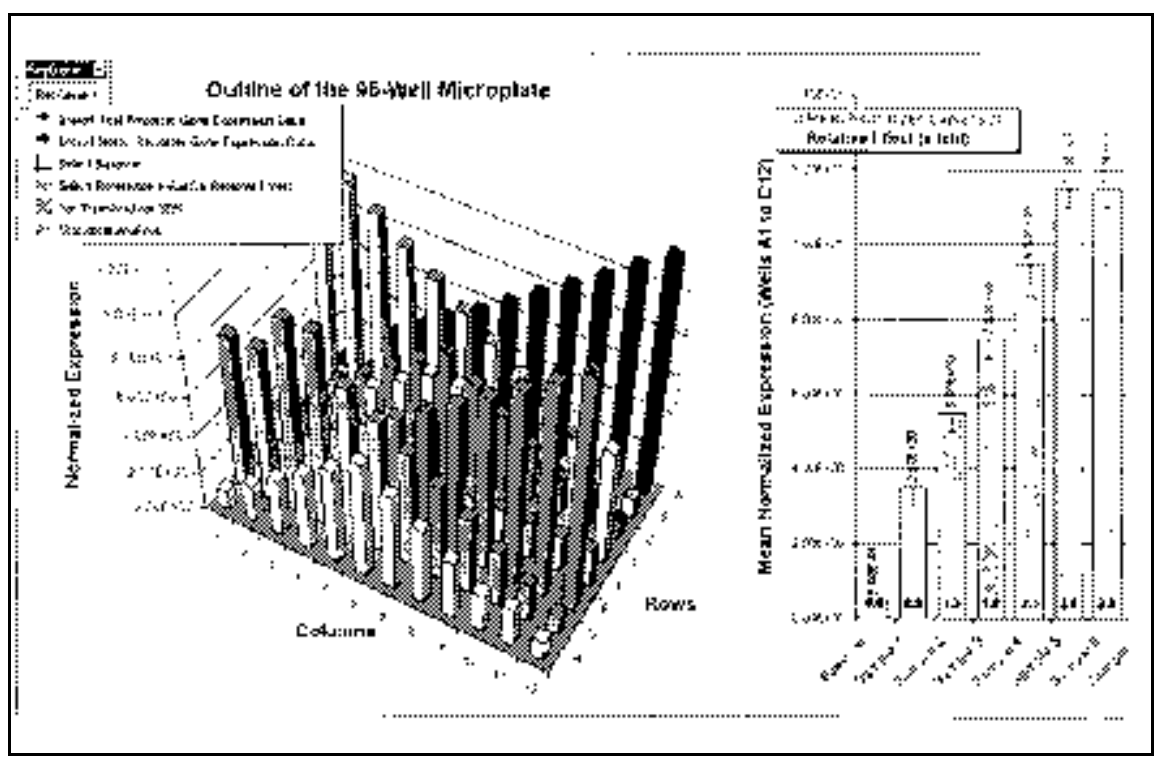

Figure 2. Screen output of the Analysis worksheet. The 3-D chart reflects the normalized expression values in the outline of a 96-well microplate, and the 2-D chart shows the mean normalized expression values, their error bars calculated on the basis of the standard error and the relative effect. Several VBA programs, which can be launched by the menu items from the RepGene toolbar, will assist the user in managing essential procedures, such as data import and statistical analysis. 


\section{BoCompuling}

matic comparison of data across experiments is limited. RepGene is not meant to replace the use of a database application if sophisticated comparisons of expression values across experiments are needed. In this case, the expression values can be easily exported into any database application, as they are arranged in distinct columns.

The RepGene file referred to in this article can be obtained free of charge from the authors. Moreover, the VBA source code of the modules and forms can be edited and extended to modify or to develop VBA applications.

\section{ACKNOWLEDGMENTS}

The authors thank Etienne Studer for his expertise and his helpful advice concerning the VBA source code. Moreover, the authors thank the colleagues who assisted them with their

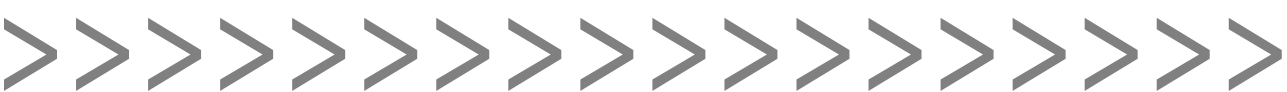

valuable experience and discussions in the field of reporter gene assays. This work was supported by the Swiss National Science Foundation grant no. 3200-049125.96/1. A.R.M. is supported by the Swiss Clinicians Opting for REsearch (SCORE A) grant no. 3231048896.96 of the Swiss National Science Foundation.

\section{REFERENCES}

1.Alam, J. and J.L. Cook. 1990. Reporter genes: application to the study of mammalian gene transcription. Anal. Biochem. 188:245254.

2.Broach, J.R. and J. Thorner. 1996. Highthroughput screening for drug discovery. Nature 384:14-16.

3.Bronstein, I., J. Fortin, P.E. Stanley, G.S. Stewart, and L.J. Kricka. 1994. Chemiluminescent and bioluminescent reporter gene assays. Anal. Biochem. 219:169-181.

4.Felgner, J.H., R. Kumar, C.N. Sridhar, C.J. Wheeler, Y.J. Tsai, R. Border, P. Ramsey, M. Martin et al. 1994. Enhanced gene deliv- ery and mechanism studies with a novel series of cationic lipid formulations. J. Biol. Chem. 269:2550-2561.

5.Karin, M. 1994. Signal transduction from the cell surface to the nucleus through the phosphorylation of transcription factors. Curr. Opin. Cell Biol. 6:415-424.

6.Lorenz, W.W., M.J. Cormier, D.J. O'Kane, D. Hua, A.A. Escher, and A.A. Szalay. 1996. Expression of the Renilla reniformis luciferase gene in mammalian cells. J. Biolumin. Chemilumin. 11:31-37.

7.Montminy, M. 1997. Transcriptional regulation by cyclic AMP. Annu. Rev. Biochem. 66:807-822.

Received 1 December 2000; accepted 25 April 2001.

Address correspondence to:

PD Dr. André R. Miserez

Head Cardiovascular Genetics

Coordinator Human Molecular Genetics Groups Department of Clinical-Biological Sciences University of Basel

Hebelstrasse 20, CH-4031 Basel, Switzerland e-mail: andre-r.miserez@unibas.ch 